\title{
Is the association between irritable bowel syndrome and abuse explained by neuroticism? A population based study
}

\author{
N J Talley, P M Boyce, $M$ Jones
}

\begin{abstract}
Background-In outpatients and the community, an association between abuse (particularly sexual abuse) and irritable bowel syndrome (IBS) has been observed, but whether there is a causal link continues to be disputed.
\end{abstract}

Aims-To test the hypothesis that psychological factors explain the apparent association between abuse and IBS.

Methods-A sample of residents of Penrith (a Sydney suburb sociodemographically similar to the Australian population) selected randomly from the electoral rolls (that by law include the entire population of age 18 years and above) was mailed a validated self report questionnaire. Measured were gastrointestinal (GI) symptoms including the Rome criteria for IBS, abuse (including the standardised Drossman questions), neuroticism (Eysenck Personality Questionnaire), and psychological morbidity (General Health Questionnaire).

Results-The response rate was $64 \%$ $(n=730) ; 12 \%$ fulfilled the Rome criteria for IBS. Overall abuse in childhood (odds ratio $(O R)=2.02,95 \%$ confidence interval (CI) 1.29 to 3.15 ) but not adulthood (OR=1.39, 95\% CI 0.88 to 2.19) was associazted with IBS univariately. Neuroticism and psychological morbidity were also univariately associated with abuse in childhood, abuse in adulthood, and IBS, respectively. However, by logistic regression, abuse in childhood was not associated with IBS after controlling for age, gender, and psychological factors $(\mathrm{OR}=1.34,95 \%$ CI 0.83 to 2.17$)$. The results were not altered by restricting the analyses to more severe forms of abuse, and were not explained by interactions between abuse and psychological variables.

Conclusion-There is an association between abuse and IBS in the community, but this may be explained in part by other psychological factors. Based on a path analysis, we postulate that abuse may induce the expression of neuroticism that in turn leads to IBS.

(Gut 1998;42:47-53)

Keywords: epidemiology; irritable bowel syndrome; abuse; neuroticism
Irritable bowel syndrome (IBS) is a common and costly condition. ${ }^{12}$ Management of IBS remains unsatisfactory because the aetiology is obscure. Psychological factors and psychiatric disorders have been implicated in the pathogenesis but this remains controversial..$^{3-5}$ Most studies have focused only on those who have consulted; two separate volunteer studies reported that psychological factors were associated with health care seeking by IBS sufferers rather than IBS symptoms per se, suggesting that psychological distress is not causally related to IBS. ${ }^{67}$ However, careful population based studies that have applied strict criteria for the identification of IBS have not investigated the association between psychological factors and IBS.

Since Drossman reported in female outpatients that a past history of sexual and physical abuse was associated with IBS, ${ }^{8}$ there has been increasing interest in this relationship. We have shown that a history of sexual abuse is linked to IBS symptoms in the general population. ${ }^{9}$ We and others have also confirmed that, in outpatients, a history of abuse is associated with IBS and may contribute to a poor outcome. ${ }^{10-12}$ However, it remains unclear whether the association of abuse with IBS reflects a causal relationship or whether other factors such as personality traits or psychiatric comorbidity explain the observations.

We have previously hypothesised that some individuals in the population may be "positive reporters" who are disposed to interpret normal somatic sensations as pathological. ${ }^{13}$ If this is true, then the association of abuse with IBS may be explained by underlying personality traits. We wished to test this hypothesis in a population based study. Our goal was to assess the association between IBS, abuse (particularly sexual abuse), the personality trait neuroticism, and current psychological morbidity.

\section{Methods}

QUESTIONNAIRE

The Bowel Symptom Questionnaire (BSQ) has been previously validated in Australia. ${ }^{14}$ The BSQ is a modified version of the previously validated and widely used Bowel Disease Questionnaire. ${ }^{15}$ It incorporates questions which identify IBS using the new standard, the Rome criteria. ${ }^{16}$

We included in the BSQ two validated psychological questionnaires. The General Health Questionnaire (GHQ) was developed for population surveys, ${ }^{17}$ specifically to detect
Accepted for publication 29 May 1997 


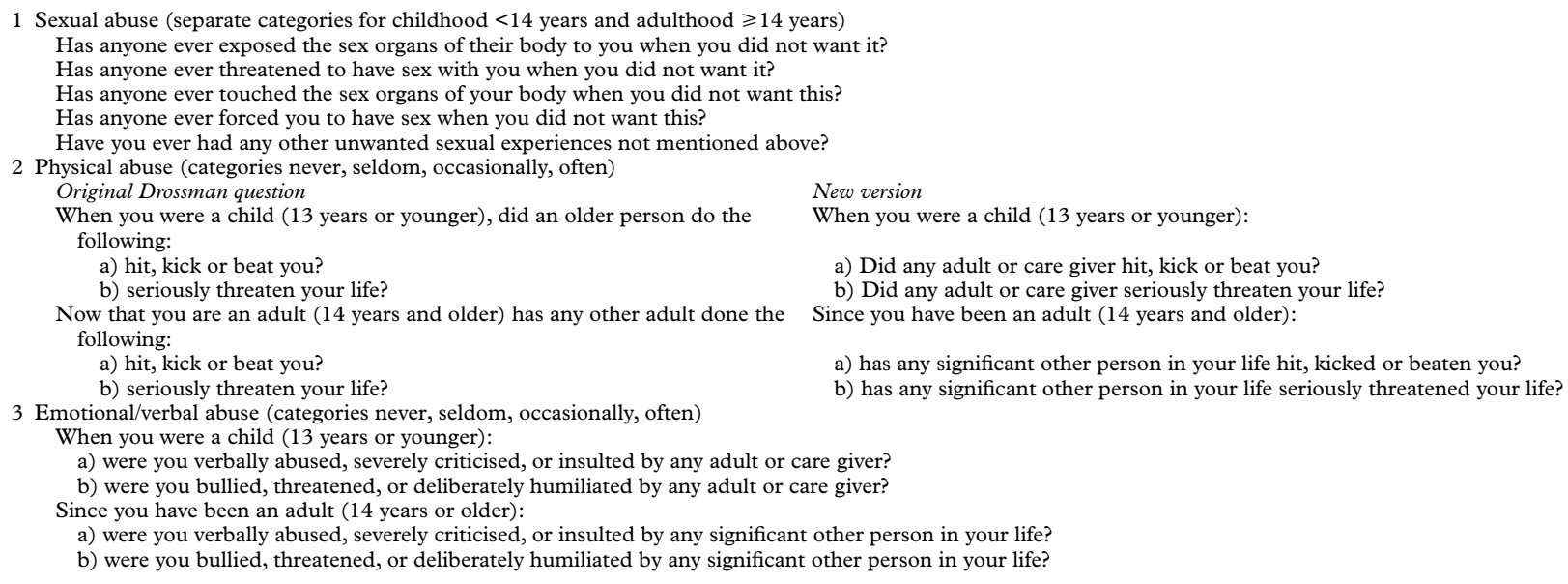

non-psychotic psychiatric illness (anxiety and depression). The GHQ is a state measure of psychological morbidity (assessing features in the prior few weeks); it does not provide a specific psychiatric diagnosis. The Eysenck Personality Questionnaire (short neuroticism subscale) measures the personality trait neuroticism which we hypothesised could be a key vulnerability factor in IBS. ${ }^{18}$

We included the sexual abuse questions validated by Drossman et $a l^{819}$ that we have applied in a US study of abuse and IBS. ${ }^{9}$ The Drossman physical abuse questions were included in addition, with some minor modifications (table 1). Minor changes in grammar and format were made to ensure comprehension by the local population. The referent terms used to identify the perpetrator in the questions relating to childhood abuse were changed to eliminate positive responses to minor events such as fights at school or bullying. While it is conceded that a child could experience some long term consequences from such incidents if they were repetitive, frequent, or severe, these events are usually qualitatively distinct from child abuse as it is generally understood. ${ }^{19}$ In the case of older siblings, it was our decision that for the purposes of this study, any violent behaviours by older siblings would be considered "abuse" only if they were in the position of being a care giver for the child. This decision was taken because in such a brief, self report format it is not possible to discover the minority of cases where violence from older siblings or school friends occurred in circumstances sufficient to classify as abuse. Similarly, the change of wording for adult physical abuse was made to eliminate positive responses on the basis of one off events (for example, mugging) or occasional violence from strangers or acquaintances (for example, crime related or job related), since these events are outside the realm of abuse as conceptualised by our research definition. Additionally, we incorporated four questions to assess emotional/verbal abuse based on our previous population based study. ${ }^{9}$ These questions have not yet been formally validated. However, they have excellent face validity, and represent a dimension of abuse which has been little studied in relation to gastrointestinal disorders.

Response options for the emotional/verbal abuse questions were identical to those used in the Drossman physical abuse questions (never, seldom, occasionally, or often). Subjects endorsing physical or emotional/verbal abuse occasionally or often were considered to have suffered abuse to increase the sensitivity of the survey, but the primary analysis considered the often categories alone.

SUBJECTS

A total of 1500 subjects was randomly selected from the Electorate of Lindsay list of voters. ${ }^{20}$ This area, which includes Penrith, Australia, and surrounding suburbs, has a population (in 1993) of 73393 (3.6\% of the Sydney population). This population is demographically very similar to the Australian population according to 1991 census data, except that it is slightly younger and has a slightly higher socioeconomic status. ${ }^{14} 20$ The list of selected subjects was divided into three batches of 500 subjects, and mailings of the BSQ to them were begun at intervals approximately two months apart, for ease of administration.

Subjects were offered a $\$ 2.00$ lottery ticket in return for completing the questionnaire. Subjects were given the option of refusing. Reminder letters were sent at two week intervals. At week 6 this included another questionnaire. At week 10, we attempted to telephone subjects who had not yet responded. Finally, the survey was closed at 12 weeks.

Of the 1500 people in the general community to whom we sent the questionnaire, $323(22 \%)$ had moved, and were excluded on that basis. Another 42 (3\%) were excluded due to serious illness, death, or language difficulties, leaving us with a valid sample receiving the questionnaire of 1135 subjects. By the close of the survey at the beginning of November 1995, a completed questionnaire was returned by 730 subjects, giving a response rate of $64 \%$.

\section{DEFINITION OF IBS}

The Rome criteria were applied to identify IBS subjects. ${ }^{16}$ IBS was defined as abdominal pain 
Table 2 Prevalence of abuse among Sydney adult residents

\begin{tabular}{lccc}
\hline Abuse & Women $(n=390)$ & Men (n=336) & Overall $(n=726)$ \\
\hline Childhood sexual abuse & $26.5(22.1$ to 30.8$)$ & $13.5(9.8$ to 17.1$)$ & $20.1(17.2$ to 23.0$)$ \\
$\quad$ Physical abuse & $18.5(14.7$ to 22.4$)$ & $17.2(13.2$ to 21.2$)$ & $17.9(15.1$ to 20.6$)$ \\
Often & $6.8(4.3$ to 9.2$)$ & $3.0(1.2$ to 4.9$)$ & $4.9(3.4$ to 6.5$)$ \\
Emotional/verbal abuse & $29.3(24.8$ to 33.8$)$ & $20.6(16.3$ to 24.9$)$ & $25.0(21.9$ to 28.2$)$ \\
Often & $11.0(7.9$ to 14.1$)$ & $5.5(3.0$ to 7.9$)$ & $8.3(6.3$ to 10.3$)$ \\
Adulthood sexual abuse & $21.2(17.2$ to 25.3$)$ & $6.7(4.1$ to 9.4$)$ & $14.1(11.6$ to 16.7$)$ \\
Physical abuse & $10.7(7.6$ to 13.8$)$ & $3.6(1.6$ to 5.6$)$ & $7.2(5.3$ to 9.1$)$ \\
Often & $4.1(2.1$ to 6.0$)$ & $0.2(0$ to 0.7$)$ & $2.2(1.1$ to 3.3$)$ \\
Emotional/verbal abuse & $27.4(23.0$ to 31.9$)$ & $16.6(12.6$ to 20.6$)$ & $22.1(19.1$ to 25.1$)$ \\
Often & $10.6(7.5$ to 13.6$)$ & $1.4(0.2$ to 2.7$)$ & $6.1(4.4$ to 7.8$)$ \\
\hline
\end{tabular}

Data represent prevalence per 100. The prevalence rates in women and men were directly age adjusted to the Australian population structure in 1991. The overall prevalence rates were directly age and sex adjusted to the Australian population structure in 1991. Inadequate data on four subjects are not included.

^Occasionally or often.

that kept recurring over a period of three months or more in the previous year, in combination with:

(1) One or more of the following symptoms: pain relieved by defaecation, often (more than $25 \%$ of the time); more and/or less bowel movements with pain, often; looser and/or harder stools with pain, often; and

(2) Two or more of the following: more than three bowel movements a day and/or less than three bowel movements a week, often; stools very lumpy or hard and/or stools very loose or watery, often; incomplete rectal evacuation and/or urgency and/or straining, often; abdominal bloating or distension, often; and/or mucus in stools.

STATISTICAL ANALYSIS

The survey design aimed to be able to estimate the prevalence of any gastrointestinal symptom to within five percentage points with $95 \%$ confidence. It was anticipated that all symptoms of interest would have a prevalence of less than $30 \%$. To have no more than a five percentage point error around a prevalence estimate of $30 \%$ requires a little under 300 subjects. Since it was further desired to be able to estimate such rates with the same precision in males and females, a total sample of $300 \times 2=600$ was sought. The sample actually mailed ensured that at least this number was available for analysis.

The prevalence of abuse was estimated using the observed age and gender specific proportions of affected subjects in our sample. Age adjusted and overall age and gender adjusted prevalence rates were obtained by directly adjusting the observed proportions to the demographic characteristics of the 1991 Australian population aged 18 and over. Ninety five per cent confidence intervals (CI) were calculated based on the binomial distribution. Logistic regression analyses were used to assess the association between patient characteristics and IBS or abuse, after controlling for potential confounders.

Intergroup comparisons of discrete characteristics used the Pearson $\chi^{2}$ test while comparisons of numeric characteristics, such as the neuroticism score, used the nonparametric Wilcoxon rank sum test. Correlation analysis was similarly non-parametric, using the Spearman rank correlation.

The exploration among multiple abuse variables used backward stepwise selection. The estimated odds ratios (OR) and 95\% confidence intervals were computed via logistic regression. The possibility that the effect of abuse on IBS was modified by other psychological variables was examined through interaction terms involving abuse and the variables in logistic regression models. To assess the extent to which postulated causal pathways were consistent with the data, structural equations were fitted. Results are reported as standardised coefficients $(\beta)$ and p values. All $\mathrm{p}$ values calculated were two tailed; the alpha level of significance was set at 0.05 . All numeric variables in the structural equation analysis were standardised. The coefficients $(\beta)$ represent a standardised measure (unitless) of effect of the specified independent variable on the specified dependent variable.

Table 3 Sexual abuse in subjects with and without irritable bowel syndrome (IBS)

\begin{tabular}{lccl}
\hline Abuse & No IBS \% (n) & IBS \% (n) & Odds ratio (95\% CI) \\
\hline Childhood exposure sex organ & $14.9(612)$ & $18.2(85)$ & $1.33(0.74$ to 2.39$)$ \\
Childhood threat of sex & $4.9(614)$ & $6.1(82)$ & $1.26(0.48$ to 3.36$)$ \\
Childhood sex organ touched & $10.9(614)$ & $14.3(84)$ & $1.36(0.70$ to 2.64$)$ \\
Childhood touch others & $6.4(614)$ & $6.0(83)$ & $0.95(0.36$ to 2.47$)$ \\
Childhood rape & $3.1(614)$ & $2.4(83)$ & $0.77(0.18$ to 3.38$)$ \\
Childhood other sex experience & $2.6(610)$ & $11.8(85)$ & $4.95^{\star}(2.17$ to 11.30$)$ \\
Any childhood sexual abuse & $20.4(616)$ & $27.1(85)$ & 1.44 (0.86 to 2.42$)$ \\
Adulthood exposure sex organ & $9.1(628)$ & $15.1(86)$ & $1.78(0.93$ to 3.42$)$ \\
Adulthood threatened with sex & $7.6(631)$ & $16.3(86)$ & $2.36^{\star}(1.24$ to 4.50$)$ \\
Adulthood sex organ touched & $8.0(629)$ & $14.9(87)$ & $2.03^{\star}(1.06$ to 3.72$)$ \\
Adulthood touch others & $3.8(631)$ & $12.6(87)$ & $3.66^{\star}(1.72$ to 7.77$)$ \\
Adulthood rape & $6.2(631)$ & $14.9(87)$ & $2.67^{\star}(1.36$ to 5.22$)$ \\
Adulthood other sex experience & $2.9(629)$ & $7.0(86)$ & $2.55(0.98$ to 6.60$)$ \\
Any adulthood sexual abuse & $14.1(631)$ & $23.0(87)$ & $1.82^{\star}(1.05$ to 3.14$)$ \\
\hline
\end{tabular}

$\mathrm{CI}$, confidence interval; $\mathrm{n}$, number responding to question. ${ }^{\star} \mathrm{p}<0.05$. 
Table 4 Physical and emotional/verbal abuse in subjects with and without irritable bowel syndrome (IBS)

\begin{tabular}{llll}
\hline Abuse & No IBS \% (n) & IBS \% (n) & Odds ratio (95\% CI) \\
\hline Childhood hit/kicked/beaten often & $4.8(629)$ & $10.1(89)$ & $2.25^{\star}(1.03$ to 4.90$)$ \\
Childhood verbal abuse often & $7.0(628)$ & $18.0(89)$ & $2.91^{\star}(1.56$ to 5.42$)$ \\
Childhood life threatened often & $1.6(629)$ & $5.6(89)$ & $3.68^{\star}(1.23$ to 11.04$)$ \\
Childhood bullied/threatened/humiliated often & $5.1(630)$ & $13.5(89)$ & $2.91^{\star}(1.44$ to 5.89$)$ \\
Adulthood hit/kicked/beaten often & $1.4(633)$ & $6.7(89)$ & $5.01^{\star}(1.74$ to 14.44$)$ \\
Adulthood verbal abuse often & $5.9(633)$ & $12.4(89)$ & $2.27^{\star}(1.11$ to 4.64$)$ \\
Adulthood life threatened often & $0.5(633)$ & $2.3(89)$ & $4.83(0.80$ to 29.30$)$ \\
Adulthood bullied/threatened/humiliated often & $2.4(633)$ & $11.2(89)$ & $5.22^{\star}(2.27$ to 12.00$)$ \\
\hline CI, confidence interval; n, number responding to question. & & \\
${ }^{*} \mathrm{p}<0.05$. &
\end{tabular}

\section{Results}

The mean age of the 730 respondents was 45.2 (SD 14.6), range 20-89 years. The present sample was composed of $54 \%$ young adults (20-44 years), $29 \%$ middle aged (45-59 years), and $17 \%$ elderly (60 years and over) subjects. This compared closely with data from the 1991 Australian census, which showed $56 \%, 22 \%$, and $22 \%$, respectively, in these age groups. Similarly, the sample was composed of $54 \%$ females compared with $50.4 \%$ in the Australian population. In terms of educational attainment, the sample was composed of $15 \%$ with tertiary qualifications, $37 \%$ who had completed secondary education (or higher), and $48 \%$ who had not completed secondary education. This is slightly lower than the Australian population where the corresponding findings were $16 \%, 53 \%$, and $31 \%$, respectively. The present sample was composed of $71 \%$ married subjects, $17 \%$ separated/divorced/widowed subjects, and $12 \%$ who had never been married. There was a slightly higher trend towards being married than the Australian population, where the corresponding figures were $56 \%, 15 \%$, and $29 \%$, respectively.

Overall, the sample appears to be demographically similar to the general Australian population and shows no clear bias towards local or atypical features.

IRRITABLE BOWEL SYNDROME

A total of 90 subjects $(12 \%, 95 \%$ CI 10 to 15$)$ fulfilled the Rome criteria for IBS, and this syndrome was more common in women $(17 \%$, $95 \%$ CI 13 to 21$)$ than in men $(7 \%, 95 \%$ CI 5 to $11 ; \mathrm{p}<0.001)$. There was no significant association between age and IBS. IBS was associated with lower socioeconomic status based on educational levels $(\mathrm{p}<0.01)$.

PREVALENCE OF ABUSE

Sexual, physical, and emotional/verbal abuse were common in the community. Table 2 documents the prevalence of the different types of abuse. Tables 3 and 4 present the frequency

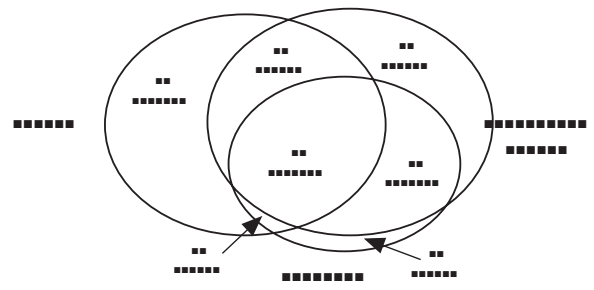

Figure 1 The overlap between sexual, physical, and emotional/verbal abuse in Sydney, Australia. The percentages represent sample prevalence in the community. of sexual and other forms of abuse in subjects with and without IBS. All types of abuse (sexual, physical, and emotional/verbal abuse) were reported more often by women than men (all $\mathrm{p}<0.05)$, and did not differ among subjects with different educational levels (all $\mathrm{p}>0.1$ ). Sexual abuse was more likely to be reported by younger subjects $(p<0.001)$. All types of abuse were reported less commonly among currently married subjects and more commonly among separated subjects, after adjusting for age and gender $($ all $\mathrm{p}<0.05)$. Figure 1 shows the overlap of abuse categories.

CORRELATION OF ABUSE VARIABLES

The separate items within the sexual, physical, and emotional/verbal abuse categories were generally moderately or strongly intercorrelated with each other based on Spearman rank correlations. Among the sexual abuse items, all correlations were significant among the childhood and adulthood abuse items, ranging from 0.10 to 0.62 . Similarly, in childhood and adulthood the physical and emotional/verbal item correlations were all significant and ranged from 0.24 to 0.61 .

ABUSE AND PSYCHOLOGICAL FACTORS

Overall, abuse in childhood (yes/no) was associated with neuroticism $(\mathrm{OR}=1.14$ per point on the 10 point neuroticism scale, $95 \%$ CI 1.08 to 1.20) and separately with psychological morbidity ( $O R=1.10$ per point on the 12 point GHQ scale, 95\% CI 1.07 to 1.14 ). Similarly, abuse in adulthood was associated with neuroticism $(1.20,95 \%$ CI 1.13 to 1.26$)$ and also with psychological morbidity $(\mathrm{OR}=1.19,95 \%$ CI 1.13 to 1.25 ).

All except two of the sexual abuse items (threat of sex in childhood and other unwanted sexual experiences in adulthood) were individually associated with neuroticism. Similarly, all of the sexual abuse items were individually associated with psychological morbidity The physical and emotional/verbal items were also associated with neuroticism (except for childhood physical abuse) and psychological morbidity (all items) (all $\mathrm{p}<0.05)$.

INDIVIDUAL ABUSE ITEMS AND IRRITABLE BOWEL SYNDROME

The individual childhood sexual abuse measures univariately were not associated with IBS except for the variable labelled "other unwanted sexual experiences" (OR=4.95, table 3). In a multivariate analysis, after controlling for other unwanted sexual experiences as well as age, gender, neuroticism, and psychological 
Table 5 Distribution of age and psychological factors in subjects with and without irritable bowel syndrome (IBS)

\begin{tabular}{lcccccccc}
\hline & \multicolumn{3}{l}{ No IBS $(n=641)$} & & \multicolumn{2}{l}{ IBS $(n=89)$} \\
\cline { 2 - 3 } Variable & Median & $25 \%$ & $75 \%$ & & Median & $25 \%$ & $75 \%$ \\
\hline Age (y) & 43 & 35 & 54 & & 45 & 34 & 55 \\
Neuroticism (score) & 3.0 & 1.0 & 5.0 & & 5.0 & 4.0 & 8.0 \\
Psychological morbidity (score) & 0.0 & 0.0 & 2.0 & & 2.0 & 1.0 & 6.0 \\
Gender (\% female) & 51.1 & & & & 72.2 & & \\
\hline
\end{tabular}

25 th and 75 th percentiles of the distribution.

morbidity, no other items added significantly to the explanation of IBS (OR=3.71, 95\% CI 1.42 to 9.67$)$.

Univariate analyses indicated that all sexual abuse items in adulthood except for unwanted exposure of the sex organs and other unspecified experiences were associated with IBS, with odds ratios ranging from 1.8 to 3.7 (table 3). On multivariate analysis, no adult abuse items significantly contributed to explaining IBS in the model adjusting for age, gender, neuroticism, and psychological morbidity.

All physical and emotional/verbal abuse forms in childhood were associated with IBS (table 4). In a multivariate analysis, after controlling for verbal abuse $(\mathrm{OR}=2.90 ; 95 \%$ CI 1.56 to 5.40), none of the other childhood emotional/physical abuse items contributed significantly to IBS.

The physical and emotional/verbal abuse items in adulthood were all significantly associated with IBS as shown in table 4 , with the exception of threat to life. After controlling for being bullied or threatened $(\mathrm{OR}=5.59,95 \% \mathrm{CI}$ 2.40 to 13.00 ), no other items contributed significantly to IBS on multivariate analysis.

IRRITABLE BOWEL SYNDROME AND

PSYCHOLOGICAL FACTORS

Subjects with IBS reported higher neuroticism scores $(p<0.001)$, higher psychological morbidity scores $(p<0.001)$, and were more likely to be female $(p<0.001)$. Age was not significantly associated with IBS (table 5).

RELATIONSHIP BETWEEN ABUSE AND IBS CONTROLLING FOR PSYCHOLOGICAL FACTORS Logistic regression analysis was used to determine the relationship between IBS and any abuse in childhood and separately in adulthood after controlling for age, gender, neuroticism,

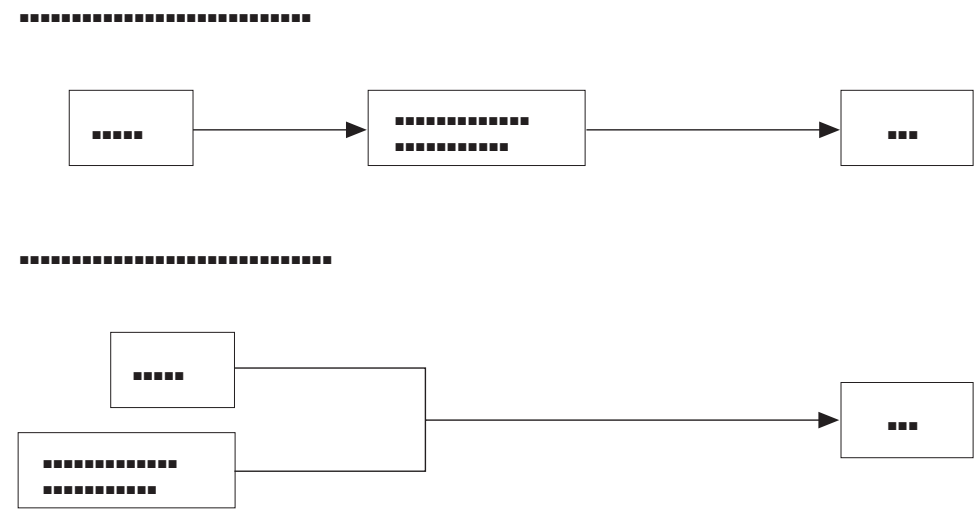

Figure 2 Two possible causal models explaining the association of abuse and psychological disturbance in IBS. and psychological morbidity. While childhood abuse overall was associated with IBS (OR=2.02, 95\% CI 1.29 to 3.15 ) on its own, this lost significance when the other factors were controlled for in the logistic regression analysis (childhood abuse OR=1.34, 95\% CI 0.83 to 2.17 ). Adulthood abuse overall was not associated with IBS on its own (OR=1.39, $95 \%$ CI 0.88 to 2.19) or when other factors were controlled for $(\mathrm{OR}=0.75,95 \%$ CI 0.44 to 1.25).

Sexual abuse was separately evaluated controlling for other factors. Childhood sexual abuse was not associated with IBS on its own $(\mathrm{OR}=1.44,95 \%$ CI 0.86 to 2.42$)$ or when other factors were controlled for $(\mathrm{OR}=0.90$, $95 \%$ CI 0.51 to 1.58$)$. On the other hand, a global rating of adulthood sexual abuse was associated with IBS on its own (OR $=1.82,95 \%$ CI 1.05 to 3.14 ), but in a model adjusting for psychological and demographic variables adulthood sexual abuse was no longer significant (OR=1.04, 95\% CI 0.57 to 1.90$)$.

To address the possibility that the effect of abuse as reported above was diluted by the inclusion of relatively "mild" forms, the analysis was repeated by restriction to the most severe forms - childhood and adult rape, and threat to life. In none of these forms of abuse was a statistically significant effect found after controlling for age, gender, neuroticism, and psychological morbidity.

The possibility that sexual abuse increases the prevalence of IBS within a subset of the population was examined through an analysis of effect modification. Possible effect modifying variables for abuse were gender and other psychological measures. Sexual abuse was defined as any childhood or any adult sexual abuse as well as childhood rape or adult rape (the most severe form). There was, however, no evidence that the effect of the abuse on the prevalence of IBS was modified by any of the variables analysed.

\section{POTENTIAL CAUSAL PATHWAYS}

One of the most difficult issues to resolve from the results is how to interpret the abuse, neuroticism, IBS triangle. The relationship between abuse and IBS was not orthogonal to the relationship between neuroticism and IBS. Does this, however, represent a confounding relationship or an intervening variable, and if the latter, which comes first? A plausible causal model postulates that childhood and adulthood sexual abuse lead to neuroticism as measured at the time of study and that this psychological disturbance is related to the development of IBS (fig 2). We have therefore assessed the extent to which the data support this purported causal pathway using structural equations (path analysis). These showed that any childhood sexual abuse $(\beta=0.10, p=0.01)$ and adult sexual abuse $(\beta=0.51, p<0.0001)$ affected levels of neuroticism and psychological morbidity $(\beta=0.20, p<0.0001$ and $\beta=0.62, p=0.0005$, respectively) but not IBS. Similarly, neuroticism $(\beta=0.14, \quad p=0.0002)$ and gender $(\beta=0.06$, $\mathrm{p}=0.045)$ but not the other psychological 
factors independently affected the prevalence of IBS, supporting model 1 in fig 2 .

\section{Discussion}

The relationship between IBS and abuse remains contentious, although a causal link has been postulated. ${ }^{19}$ We have explored this issue in a community study for the first time. To establish a causal path between sexual abuse, psychological disturbance, and IBS would require a prospective cohort study of thousands of individuals with a several decade long follow up period. In such a study the temporal ordering of events could be established and cause and effect inferred with confidence. In the absence of longitudinal studies, cross sectional studies, such as the current investigation, attempt to approximate longitudinal measurements with cross sectional measurements which ask about timing of past events. Two main causal models of abuse appear to be in contention (fig 2).

Our results suggest that while abuse is associated with IBS, the link with abuse seems to be largely explained by psychological factors. Neuroticism is a personality trait characterised by exaggerated responsivity to physiological changes. ${ }^{22}$ It has been found to be a vulnerability factor for non-psychotic psychiatric disorders and it may manifest as physical symptoms in the absence of structural disease. There is also evidence that neuroticism is largely genetically determined. ${ }^{21}$ We found that abuse was associated with neuroticism and psychological morbidity, which is consistent with other data. ${ }^{19}$ On the other hand, IBS was strongly associated with neuroticism and psychological morbidity as well as a number of sexual and emotional abuse items. However, after we controlled for neuroticism and psychological morbidity, there was no significant association between childhood abuse overall and IBS. Similarly, sexual abuse in adulthood was no longer associated with IBS after allowing for these other factors. No subgroup effects were identified by examining interactions between abuse and gender, or between abuse and the psychological variables. Moreover, restricting the analysis to more severe forms of abuse did not alter the conclusions.

In our previous population based study which showed an association between abuse and IBS, we did not measure and control for personality factors or psychopathology, although we did adjust for social support and for state anxiety and depression using the Brief Symptom Inventory. ${ }^{9}$ We propose that neuroticism predisposes to the reporting or development of IBS symptoms by a subset of subjects. Although there is a genetic link to neuroticism ${ }^{21}$ it is conceivable that abuse is a cofactor leading to the expression of neuroticism (fig 2). This model is supported by our path analysis, a mode of analysis that tests the appropriateness of the direction of alternative causal relationships, so less probable causal pathways can be eliminated. This support should, however, be viewed as tentative pending further theoretical development and focused empirical studies as other causal paths could presumably be postu- lated. The results cannot definitively distinguish among all the possible inter-relationships between abuse and neuroticism.

Psychometric studies of outpatients have consistently reported that anxiety and depression are higher in patients with IBS. ${ }^{22}$ Chaudhary and Truelove first reported that "obsessional worrying about many trivial problems" appeared to be linked to both the onset and exacerbation of IBS symptoms. ${ }^{23}$ Latimer et al found that patients with IBS had neuroticism levels that were similar to those found in psychiatric patients. ${ }^{24}$ Nevertheless, it has been suggested that the apparent link between psychological factors and IBS is explained by selection bias, as more psychologically disturbed persons tend to present for care. ${ }^{67}$ The present population based study should have avoided this problem but we cannot exclude selection bias. For example, more neurotic or abused subjects may have been more likely to respond to the survey. We believe, however, that this bias is unlikely to have affected the present population based study as we had an acceptable response rate and subjects were unaware of the specific study objectives. Moreover, the prevalence of childhood sexual abuse identified in this study is consistent with the prevalence identified in two recent Australian studies. ${ }^{25}{ }^{26}$ Fleming reported that among 710 women randomly selected from the electoral rolls, $20 \%$ reported childhood sexual abuse and $2 \%$ rape, ${ }^{25}$ compared with $26.5 \%$ and $3 \%$, respectively in the present study. Mazza et al in a cross sectional survey of patients attending general practitioners reported that $28 \%$ had a history of childhood sexual abuse and over one quarter adulthood physical or emotional abuse. ${ }^{26}$ Thus, we do not believe that abuse was over represented in our population, and hence serious selection bias is unlikely. Our finding that IBS is linked to neuroticism in the general population is consistent with a recent prospective study that followed up postinfective diarrhoea patients; the neuroticism score was significantly higher in those who developed new onset IBS, suggesting that it is not chronic bowel symptoms that induce psychological distress but rather that psychological factors at least in some cases predispose to IBS. ${ }^{27}$

Abuse was reported to be very common in the population. Approximately half of the respondents endorsed some type of abuse history. There was also striking overlap among the various abuse categories (fig 1). The issue of how accurately abuse was measured therefore needs to be considered. We applied well described measures to assess abuse in the population, assessed a wide range of potential abuse experiences, and found results consistent with the observations of others. ${ }^{9112526}$ We also evaluated the severity of reported sexual abuse experiences (such as rape) as it has been shown that more severe types of abuse are associated with poorer health status. ${ }^{28}$ However, the questions may have been more broadly interpreted by the population than was intended. While the measures used to assess abuse in this study are widely accepted as valid, ${ }^{19}$ more work is needed 
to confirm their concordance with other measures and their meaning when endorsed by subjects from the population. The possibility that in some cases psychological distress may lead to some persons misattributing certain experiences as abuse needs to be formally tested. In this study, however, subjects were not asked if they had been subject to abuse, but rather were asked to identify value neutral experiences so that we believe such a bias is unlikely.

The strengths of this study include the fact that it was population based. As those who do seek care may be more psychologically disturbed as shown in studies from the USA, ${ }^{67}$ investigations confined to outpatients may be misleading when trying to unravel the link between abuse and IBS. We have been able to show that responders were similar to the Australian population, suggesting that the sample was reasonably representative and hence the results are likely to be generalisable. Moreover, we used a validated set of measures. However, detailed psychological and psychiatric measures were not applied as this was impractical. Moreover, other "buffering" variables such as the family environment, level of social support, coping strategies, and the reaction of the subject to the trauma could not be taken into account. ${ }^{29}$ Prospective studies are now needed to explore the exact relationship between psychological factors, abuse, and IBS in the population.

In conclusion, the association of abuse with IBS in the general population appears to be explained in part by neuroticism and psychological morbidity. A reasonable causal pathway supported by the data is that abuse leads to the expression of neuroticism which in turn leads to IBS, but prospective data are now required.

Supported in part by research grants from the University of Sydney (Bridging Grant), the Royal Australasian College of Physicians (Cottrell Fellowship), and the National Health and Medical Research Council of Australia.

1 Talley NJ, Gabriel SE, Harmsen WS, Zinsmeister AR, Evans RW. Medical costs in community subjects with irritable bowel syndrome. Gastroenterology 1995;109:1736-41.

2 Jones R, Lydeard S. Irritable bowel syndrome in the general population. BMf 1992;304:87-90.

3 Ford MJ. The irritable bowel syndrome. 7 Psychosom Med 1986;30:399-410.

4 Talley NJ, Phillips SF, Bruce B, Twomey CK, Zinsmeister AR, Melton LJ III. Relation among personality and symptoms in nonulcer dyspepsia and the irritable bowel toms in nonulcer dyspepsia and the irt

5 Esler MD, Goulston KJ. Levels of anxiety in colonic Esler MD, Goulston KJ. Levels of an
disorders. N Engl f Med 1973;288:16-20.

6 Drossman DA, McKee DC, Sandler RS, et al. Psychosocial factors in the irritable bowel syndrome: a multivariate study of patients and nonpatients with irritable bowel syndrome Gastroenterology 1988;95:701-8.

7 Whitehead WE, Bosmajian L, Zonderman AB, Costa PT Jr, Schuster MM. Symptoms of psychologic distress associated with irritable bowel syndrome. Gastroenterology 1988; 95:709-14.

8 Drossman DA, Leserman J, Nachman G, Li Z, Gluck H, Toomey TC. Sexual and physical abuse in women with functional or organic gastrointestinal disorders. Ann Intern Med 1990;113:828-33.

9 Talley NJ, Helgeson SL, Zinsmeister AR, Melton LJ III. Gastrointestinal tract symptoms and self reported abuse: a
population-based study. Gastroenterology 1994;107:1040-9.

10 Talley NJ, Fett SL, Zinsmeister AR. Self-reported abuse and gastrointestinal disease in outpatients: association with irritable bowel-type symptoms. Am f Gastroenterol 1995;90: 366-71

11 Longstreth GF, Wolde-Tsadik G. Irritable bowel symptoms in $\mathrm{HMO}$ examinees. Prevalence, demographic factors and clinical correlates. Dig Dis Sci 1993;38:1581-9.

12 Walker EA, Gelfand AN, Gelfand MD, Katon WJ. Psychiatric diagnoses, sexual and physical victimisation, and disability in patients with irritable bowel syndrome or inflammatory bowel disease. Psychol Med 1995;25:125967.

13 Talley NJ, Boyce P. Abuse and functional gastrointestinal disorders: what is the link and should we care? Gastroenterology 1996;110:1301-4.

14 Talley NJ, Boyce P, Owen BK, Newman P, Paterson K. Initial validation of a bowel symptom questionnaire and measurement of chronic gastrointestinal symptoms in Australians. Aust NZ F Med 1995;25:302-8.

15 Talley NJ, Phillips SF, Melton LJ III, Wiltgen C, Zinsmeister AR. A patient questionnaire to identify bowel disease. Ann Intern Med 1989;111:671-4.

16 Drossman DA, Richter JE, Talley NJ, Thompson WG, Corazziari E, Whitehead WE (eds). The functional gastrointestinal disorders. Boston: Little Brown, 1994.

17 Goldberg DP. The detection of psychiatric illness by questionnaire. London: Oxford University Press, 1972.

18 Grayson DA. Latent trait analysis of the Eysenck Personality Questionnaire. F Psychiatry Res 1986;20:217-35.

9 Drossman DA, Talley NJ, Leserman J, Olden KJ, Barreiro $M$. Sexual and physical abuse and gastrointestinal illness: review and recommendations. Ann Intern Med 1995;123: $782-94$.

20 Australian Bureau of Statistics. 1991 Census of population and housing, basic community profile: Area 6350 Penrith (C), ABS catalogue No. 2722.1

21 Floderus-Myrhed B, Pedersen N, Rasmuson I. Assessment of heritability for personality, based on a short-form of the Eysenck Personality Inventory: a study of 12,898 twin pysenck Personality Inventory: a

22 Langeluddecke PM. Psychological aspects of irritable bowel syndrome. Aust NZ f Psychiatry 1985;19:218-26.

23 Chaudhary NA, Truelove SC. The irritable colon syndrome. A study of the clinical features, predisposing causes, and prognosis in 130 cases. $Q \mathcal{F} M e d$ 1962;123:307-22.

24 Latimer P, Sarna S, Campbell D, Latimer M, Waterfall W, Daniel EE. Colonic motor and myoelectric activity: a comparative study of normal subjects, psychoneurotic patients, and patients with irritable bowel syndrome. Gastroenterology 1981;80:893-901.

25 Fleming JM. Prevalence of childhood sexual abuse in a community sample of Australian women. Med f Aust 1997; 166:65-8.

26 Mazza D, Dennerstein L, Ryan V. Physical, sexual and emotional violence against women-a general practice-based prevalence study. Med F Aust 1996;164:14-17.

27 Gwee KA, Graham JC, McKendrick MW, et al. Psychometric scores and persistence of irritable bowel after infectious diarrhoea. Lancet 1996;347:150-3.

28 Leserman J, Drossman DA, Li Z, Toomey TC, Nachman G, Glogau L. Sexual and physical abuse history in gastroenterology practice: how types of abuse impact health status. Psychosom Med 1996;58:4-15.

29 Walker EA, Katon WJ. Researching the health effects of victimisation: the next generation. Psychosom Med 1996;58: 16-17. 